\title{
Standards Development of mHealth at a Glance
}

\author{
A Tavakoli Golpayegani ${ }^{1}$; F A Parand ${ }^{2,}$; Sh Ashrafiyan ${ }^{3}$ \\ ${ }^{1}$ Assistant Professor, Faculty of the Institute of Electrical and Mechanical, Standard Research Institute \\ ${ }^{2}$ Assistant Professor, Faculty of Mathematics and Computer Science, Allame Tabataba'i University, Tehran, Iran \\ ${ }^{3}$ MSc Student, Faculty of Mathematics and Computer Science, Allame Tabataba'i University, Tehran, Iran \\ * Corresponding author: F A Parand, Assistant Professor, Faculty of Mathematics and Computer Science Allame Tabataba'i University, Beheshti Square, Corner A. Kasir, Faculty of \\ Economics, Tehran, P. O. Box: 8875371, Iran. Tel: +98-218875371, Fax: +98-218875371, E-mail: parand@atu.ac.ir
}

Received: 11 Dec 2016

Accepted: 01 Jan 2017

Epub: 23 Feb 2017

Ppub: 15 Jan 2018

\begin{abstract}
Background: Mobile health (mHealth) refers to the use of mobile communication technology and smart devices such as mobile phones, laptops, tablets, and so on to provide healthcare solutions. This technology may be used for prevention, diagnosis, treatment or medical follow-up in healthcare area. Smart devices help health organizations in providing essential care and health information anytime and anywhere through information and telecommunications technologies. In this way, development and implementation of smart devices are rapidly progressing around the world. Recent development of smart devices, where the patients' information are stored and distributed securely, requires a robust and compatible architecture to be able to manage the mobile data effectively.

Objectives: The rapid changes in technology and adoption behaviors, jointly with the many proprietary systems that are hard to integrate, raise multi-layered interoperability issues. To support innovation and successful development of mHealth, agreement on interoperability needs to be established. This interoperability means that there must be a common understanding of the data elements, structures, and terminology used in the mobile and health IT space, therefore, this interoperability requires standards to be effective.

Methods: mHealth is a broad field and no single standards organization encompasses the entire field. There are some international standard organizations that are at the forefront of interoperable healthcare mobile standards development. These include integrating the healthcare enterprise (IHE), Health Level 7 (HL7), and international organization for standardization (ISO). IHE and HL7, in particular, are the leading standards developers for healthcare information interoperability, messaging, and architecture. The mentioned organizations are not an exhaustive list of standard organizations doing work in various e-health areas; however, they are the high-profileest organizations.

Results: This paper provides a survey, which introduced the standard bodies and organizations working specifically in e-health standards. Highlights considerable applied standards for the E-health mobile applications. It is clear that the issues identified in the development of standards have an impact on the adoption, conformance, and compliance in such a diverse range of standards. These standards ensure systems and software products that are safe, valid, and meet quality parameters according to accepted standards.

Keywords: mHealth; Developing Countries; Health Care.
\end{abstract}

